\title{
Photorefractive Simulton in Lithium Niobate
}

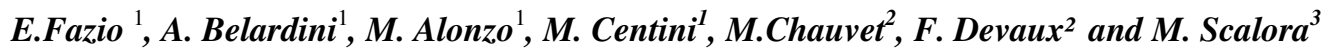 \\ 1. Ultrafast Photonics Lab, Sapienza Università di Roma, IT-00161 Roma, Italy-UE \\ 2. Département d'Optique, Institut FEMTO-ST, UMR CNRS 6174, Université de Franche-Comté, F-25030 Besançon, France-UE \\ 3. Charles M. Bowden Research Facility, RDECOM, US Army Aviation and Missile Command, Redstone Arsenal, AL-35803, USA
}

The second harmonic generation process strongly depends on the phase-matching condition of the interacting waves. Very far from perfect phase-matching, the wave coupling at the input interface of the nonlinear material generates two second-harmonic pulses, one propagating at the speed given by its refractive index, and one perfectly locked in time with the pump field, and consequently propagating exactly with the same group and phase velocities.

Such phenomenon was already theoretically previewed in 1962 [1] by J. A. Armstrong at alii and more specifically finalised in 1999 by M. Mlejnek et alii [2]. Recently W. Su et alii have experimentally confirmed the pulse twin pulse generation [3] while E. Fazio et alii [4] measured the exact locking of the two velocities.

Would it be possible to lock the fundamental and second-harmonic beam in space as well? Yes, by means of photorefractive screening solitons. Formation of bright photorefractive-photovoltaic solitons in lithium niobate was previously demonstrated by using CW laser beams [5] as well as light pulses from a Nd-Yag laser [6]. In such case the generated second-harmonic pulses slowly accumulate charges that modify the refractive index. Such modification became active for the fundamental beam as well, which consequently is self-confined, increasing its conversion efficiency.

Such process can be applied also for a second-harmonic generation process occurring at a very large phase mismatch $\left(\Delta \mathrm{k} \sim 10^{4} \mathrm{~cm}^{-1}\right)$, as for example pumping at $800 \mathrm{~nm}$ and generating second-harmonic at $400 \mathrm{~nm}$.

Taking a similar experimental configuration already used in [4], the fundamental beam was injected with an angle with respect to the normal incidence, in order to make the two second-harmonic pulse separate by means of Snell refraction. In particular the unlocked pulse (which has its own refractive index) propagates along a different direction with respect to the locked fundamental and harmonic pulses, which travel together along the same direction.

At this time an external electric field was turned on in order to allow the photorefractive screening effect to generate a modification of the refractive index and consequently to self-confine both beams (according to screening-photovoltaic soliton theory [5]).

The locked second harmonic beam self-confined, generating a waveguide that trapped the fundamental beam as well. Thus, a spatial and temporal locking of the fundamental and second-harmonic pulses was indeed obtained, because of the simultaneous occurrence of the harmonic generation and of the photorefractive nonlinearity. Thus, we call such state a photorefractive simulton. However clearly the obtained condition in not a "light bullet" in common meaning, because the spatial confinement is reached by a slowly accumulation of charges and consequently is slowly

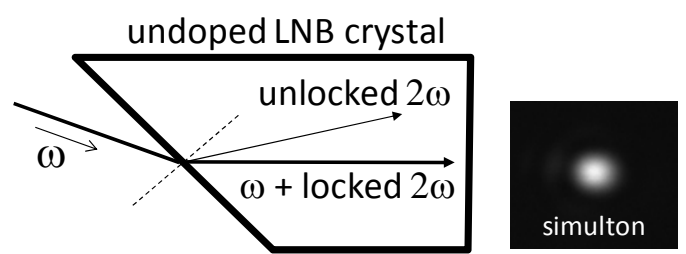

Experimental configuration for separating the two generated second harmonic pulses and the image of the fundamental and second harmonic self-confined beams at the output face of the lithium niobate crystal reached as well.

However the final result, where an almost steady state was reached, gave a perfect overlapping in time and space of the femtosecond fundamental and generated second-harmonic pulses.

\section{References}

[1] J. A. Armstrong, N. Bloembergen, J. Ducuing and P.S. Pershan, Interactions between Light Waves in a Nonlinear Dielectric, Phys. Rev. 127, 1918 (1962).

[2] M. Mlejnek, E. M. Wright, J. V. Moloney, and N. Bloembergen, Second Harmonic Generation of Femtosecond Pulses at the Boundary of a Nonlinear Dielectric, Phys. Rev. Lett. 83, 2934 (1999).

[3] W. Su, L. Qian, H. Luo, X. Fu, H. Zhu, T. Wang, K. Beckwitt, Y. Chen, and F. Wise, Induced group-velocity dispersion in phasemismatched second-harmonic generation, J. Opt. Soc. Am. B 23, 51 (2006).

[4] E. Fazio, F. Pettazzi, M. Centini, M. Chauvet, A. Belardini, M. Alonzo, C. Sibilia, M. Bertolotti, and M. Scalora, Complete pulse locking in phase-mismatched second-harmonic generation, in press on Opt. Expr.

[5] E. Fazio, F. Renzi, R. Rinaldi, M. Bertolotti, M. Chauvet, W. Ramadan, A. Petris, V.I. Vlad, Screening-photovoltaic bright solitons in lithium niobate and associated single-mode waveguides, Appl. Phys. Lett. 85, 2193 (2004).

[6] F. Pettazzi, M. Alonzo, M. Centini, A. Petris, V.I. Vlad, M. Chauvet, E. Fazio, Self-trapping of low-energy infrared femtosecond beams in lithium niobate, Phys. Rev. A 76, 063818-21 (2007). 